\title{
SYSTEMATIC LITERATURE REVIEW ON RESOURCE Allocation AND RESOURCE SCHEDULING IN Cloud Computing
}

\author{
B. Muni Lavanya and C. Shoba Bindu \\ Department of Computer Engineering, JNTUACEP, Pulivendula, AP
}

\begin{abstract}
The objective the work is intend to highlight the key features and afford finest future directions in the research community of Resource Allocation, Resource Scheduling and Resource management from 2009 to 2016. Exemplifying how research on Resource Allocation, Resource Scheduling and Resource management has progressively increased in the past decade by inspecting articles, papers from scientific and standard publications. Survey materialized in three fold process. Firstly, investigate on the amalgamation of Resource Allocation, Resource Scheduling and then proceeded with Resource management. Secondly, we performed a structural analysis on different author's prominent contributions in the form of tabulation by categories and graphical representation. Thirdly, huddle with conceptual similarity in the field and also impart a summary on all resource allocations. In cloud computing environments, there are two players: cloud providers and cloud users. On one hand, providers hold massive computing resources in their large datacenters and rent resources out to users on a per-usage basis. On the other hand, there are users who have applications with fluctuating loads and lease resources from providers to run their applications. Further, delivers conclusions by conferring future research directions in the field of cloud computing, such as reduce clouds early in the Internet, combining Resource Allocation, Resource Scheduling and Resource management rather than a Cloud model for providing high quality results, etc.
\end{abstract}

\section{KEYWORDS}

Resource Allocation, Resource Scheduling, Resource Management, Cloud Computing, SaaS, Paas, IaaS, Private Cloud, Public Cloud and Community Cloud.

\section{INTRODUCTION}

Cloud computing is a technique which provides a delivery of computing resources over the Internet. Cloud computing is one of the latest buzzwords in the world of Information Technology, which means key to the definition of cloud computing is the "cloud" itself, but, the exactly meaning of the cloud is that clouded cover computing is a set of web based computing resources that deliver on demand information services to users from any location in the world. The purpose of using cloud is that cloud is a large group of interconnected computers. These computers can be personal computers or network servers or they can be public or private. This cloud of computers extends beyond a single company or enterprise. Cloud storage is a service model in which data is maintained, managed and backed up remotely and made available to users over a network as web or typically the Internet. The applications and data served by the cloud are available to wide group of users, cross-enterprise and cross-platforms. Access is via the Internet. Any authorized user can access these documents and different applications from any computer over any Internet connection. Cloud computing transfers different computing resources over the Internet as a DOI : $10.5121 /$ ijait.2016.6401 
replacement for keeping data on user own hard disk or updating applications for user needs, to use a service over the Internet at another location, to store user information or use its applications. The cloud is web storage where your all data remain store. One can access that data from the cloud according to user requirement easily. The clouds flexibility is most useful for businesses in its ease of access, particularly with data sharing. Documents and files can be stored in the cloud and accessed via an Internet connection, which is ideal solution for businesses. Also in terms of data backup, the cloud provides peace of mind with securing useful data.

The goal of cloud computing is to concern conventional supercomputing, or high-performance computing power, normally used by military and research facilities, to perform millions of computations per second, in user-oriented applications such as financial portfolios, to deliver personalized information, to provide a large volume of data storage. Cloud computing has been changing how mostly users use the web and how they store their files of data on web. There are examples as Orkut, Facebook, Amazon and Twitter and the services like Google Docs and Gmail etc. Cloud computing technology uses networks of large group of servers typically running lowcost user PC technology with specialized connections to increase data processing tasks across them.

The resource is generally depend on computer mainframe and can also be classified into 3 layers from top to bottom: First, in the application layer - the operation status of network server, database server and other related servers, Second, Network layer - routing between hosts, data transmission bandwidth, communication data layer, Third, System Layer - CPU Information, Memory size. All these are at one side and in other hand cloud computing resources can also include operation system, disk space, network communication capabilities, data resources, equipment and other related resources.

In cloud computing, the resource allocation is also one of the processes to assign available resources for the needed cloud application users, cloud resources can be provisioned on-demand in a fine-grained, multiplexer manner. In the cloud the resource allocation is based on the software, platform and infrastructure respectively known as software as a Service(SaaS), Platform as a Service(PaaS) and Infrastructure as a service (IaaS). In cloud platforms, resource allocation takes place at two levels as follows: In first level, when the cloud application user wants to uploaded to the cloud, then the load balancer assigns the requested instances to physical computers, to balance the computational load of multiple applications across physical computers. And second level, when an application receives multiple incoming requests, these requests also should be assigned to a specific instance of application to balance the computational load across a set of instances of the same application.

Table 1.1 Search Key Terms

\begin{tabular}{|c|c|}
\hline Resource Scheduling & \multirow{2}{*}{ "ORed" } \\
\hline Resource Management & and \\
\hline Survey on Resource Allocation & "ANDed" \\
\hline Survey on Resource Scheduling & \\
\hline Survey on Resource Management & \\
\hline SLR on Resource Scheduling & \\
\hline SLR on Resource Management &
\end{tabular}


International Journal of Advanced Information Technology (IJAIT) Vol. 6, No.4, August 2016

Table 2 Quality Assessment and Data Extraction template

\begin{tabular}{|l|l|}
\hline Extracted Fields & Assessment Factors \\
\hline Author & Research Scholar / Experience \\
\hline Title & Relevant / Not Relevant \\
\hline Year & NA (Not Applicable) \\
\hline Keywords & Match / Not Match \\
\hline Objective(s)/Issue(s) & Clear / Not Clear \\
\hline Research Question(s) (optional) & Yes/No \\
\hline Framework/Model/Method/Technique/Approach & Yes/No/Other \\
\hline Pros \& Cons & Detailed / Not defined \\
\hline Case study/Empirical study/Survey & Theoretical/ Experimental/None \\
\hline Future Scope & Mentioned / Not Mentioned \\
\hline Category and Sub-category & Belongs to / Not Belongs to any \\
\hline Published in a relevant journal/conference & Yes / No \\
\hline Cited by other authors & Yes/No \\
\hline
\end{tabular}

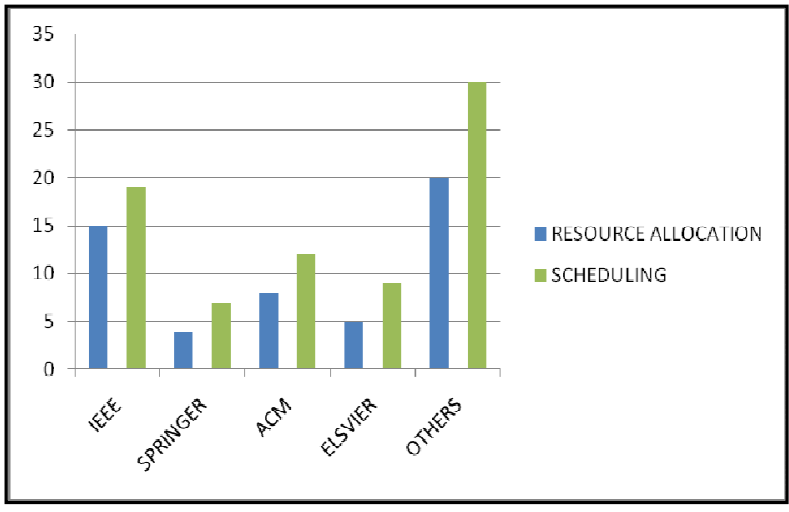

Fig. 2 Graphical representation for surveyed papers on Resource allocation and Scheduling

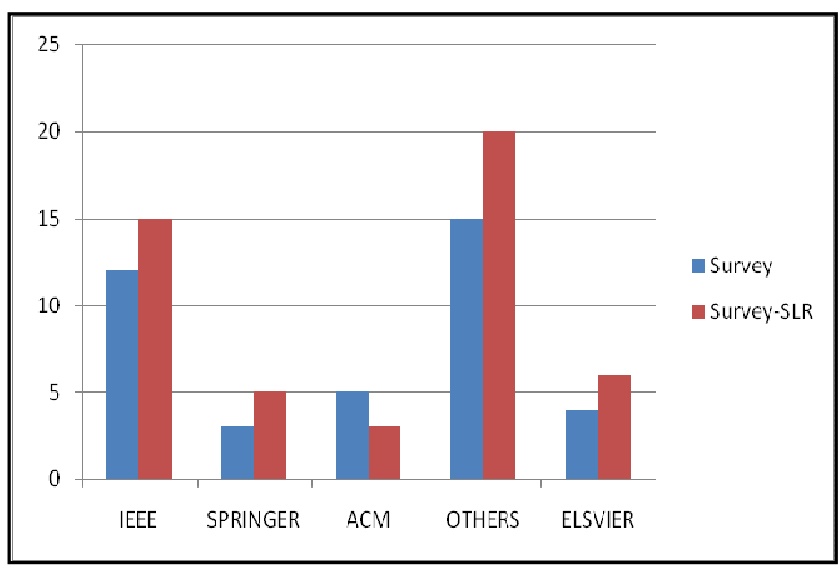

Fig. 1 Graphical representation of surveyed papers on Cloud Computing

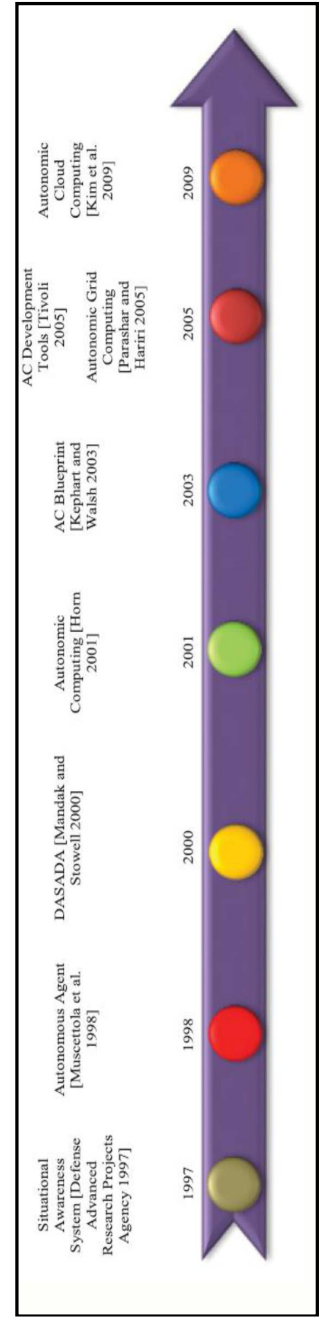

Fig. 3 Evolution of autonomic computing (Courtesv: S.Singh and I. Chana) 
In table 1 shows that search key word terms and table 2 shows the data extraction fields to assess the articles using different factors. However, an outline of survey and systematic literature review (SLR) publications is shown in figure 1 and Figure 2 shows drastically increased research on resource allocation and resource scheduling in both journal articles and conference papers from 2009 to 2016.

Next section of the paper presents as follows: section 2 speaks about literature survey search on resource allocation and resource scheduling in which different categories of article authors opinions followed by section 3 talks about in detailed on SLR, Resource allocation followed by scheduling and next section 4 gives discussion on the survey and last section 5 conclusion on resource allocation and scheduling.

\section{LITERATURE SURVEY}

\subsection{Literature Search}

A literature search identified 200 plus among which filtered to 15 conferences, 25 journal articles and Some other papers from different reputed forums, a total of both 100 articles from 2009 to 2016. Figure 2 shows the number of conference papers from standard symposium, workshops, tutorials like international conference on Cloud Computing (ICCC), Resource Allocation, Resource Scheduling and Resource management.

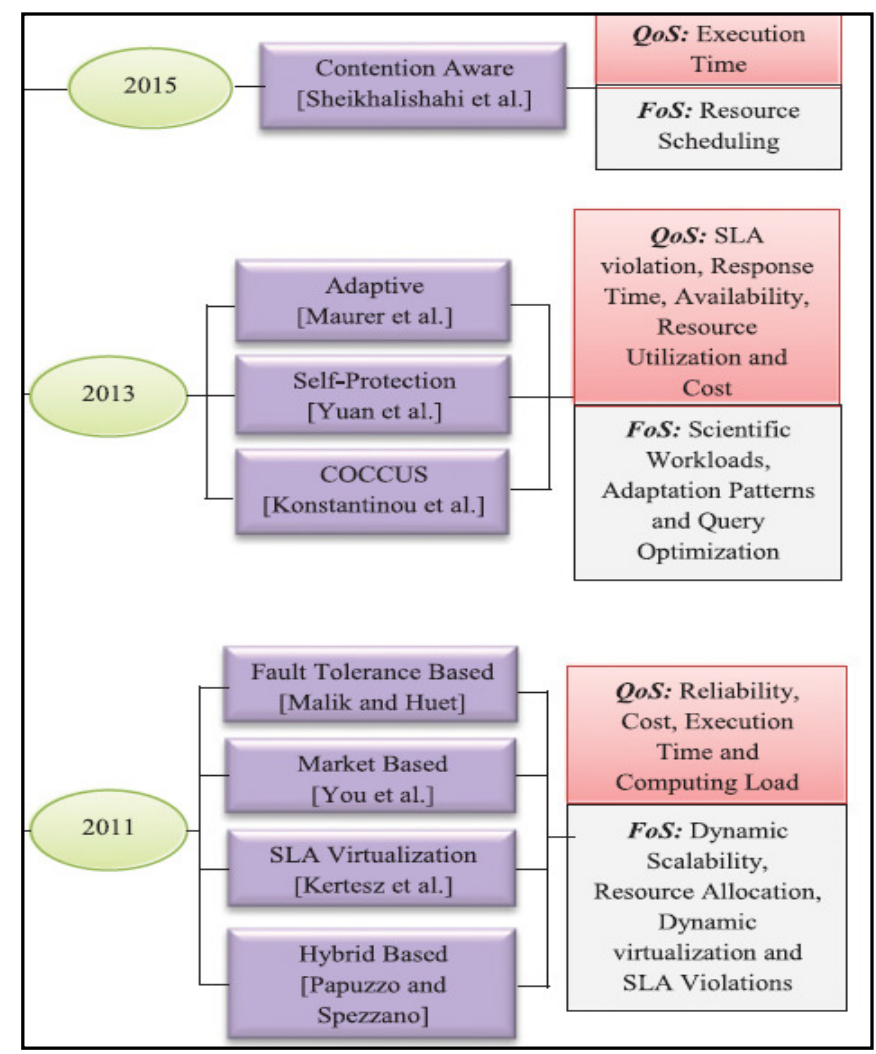

Fig. 4 Evolution of Qos in Cloud Computing 
It seems that Resource Allocation to Resource Management, Survey on Resource Allocation, Scheduling, Management and SLR on Resource Allocation, Scheduling, Management, adoption and transformation is drastically increasing and shows good attention in research communities both from academia and industry. Fig. 4 shows the evolution of quality of service in cloud computing.

\subsection{Selection Inclusion And Exclusion Criteria}

Each study that was retrieved from the manual search or an automated search is formulated as in section 2.3 and was evaluated by thesis author and his both supervisors (a total of three conductors).Divergences in the selection were work out by accord among the three conductors in the wake of examining the whole paper. The following studies were considered that met atleast one for the inclusion criteria:

- Studies presenting Resource Allocation that are used for allocating resource in cloud.

- Studies related to Resource Scheduling in cloud computing.

The following studies were excluded that met at least one from the exclusion criteria were:

- Papers that are not focused on the Resource Allocation.

- Papers presenting only ideation without any practical orientation.

- Papers presenting only Cloud computing like reusability, application-specific and flexibility.

Table 3 Different articles used in Cloud Computing Survey

\begin{tabular}{|c|c|c|c|}
\hline Category & Sub-Category & Article & Author \& Year \\
\hline \multirow[t]{2}{*}{ Survey } & $\begin{array}{l}\text { Cloud Computing } \\
\text { Issues }\end{array}$ & 9 & $\begin{array}{l}\text { Haibo Yang et al (2009) } \\
\text { Haibo Yang et al (2012) } \\
\text { Yaser Ghanam et al (2012) } \\
\text { Sunilkumar S.M et al(2013) } \\
\text { Frederico Durao et al (2014) } \\
\text { Mahmoud.M et al (2014) } \\
\text { F.F.Moghaddam et al (2015) } \\
\text { Anton Backe et al (2015) } \\
\text { Nasrin Hesabian et al (2015 }\end{array}$ \\
\hline & Scheduling & 8 & $\begin{array}{l}\text { Jaspreet kaur et al (2012) } \\
\text { Lovejit Singh et al (2013) } \\
\text { Shabnam Khan et al (2013) } \\
\text { M.Padmavathi et al (2014) } \\
\text { Sukhpal Singh et al (2015) } \\
\text { Soumen Santra et al (2015 } \\
\text { A E Mohamed Mosa (2014) } \\
\text { Malathi.P et al (2015) }\end{array}$ \\
\hline SLR & & 5 & $\begin{array}{l}\text { Yan Hu et al(2014) } \\
\text { Li Liu et al(2014) } \\
\text { Rashmi Rai et al(2015) } \\
\text { Anterpreet Kaur(2015) } \\
\text { Ilango Sriram et al(2015) }\end{array}$ \\
\hline
\end{tabular}




\subsection{Categories on cloud computing}

Table 3 shows that different articles used to study survey on cloud computing under the theme of resource allocation and resource scheduling with the fields authors and corresponding year. Similar fashion, table 4 shows exact point of articles on allocation and scheduling

Table 4 Different articles on Resource Allocation and Resource Scheduling

\begin{tabular}{|c|c|c|c|}
\hline Category & Sub-Category & Article & Author \& Year \\
\hline \multirow[t]{7}{*}{ Resource Allocation } & Resource Utilization & 7 & $\begin{array}{l}\text { Ms. Shubhangi D. et al(2012) } \\
\text { Abirami S.P et al(2012) } \\
\text { Ram Kumar Sharma et al(2013) } \\
\text { N. Asha et al (2013) } \\
\text { Xin Xu et al (2014) } \\
\text { Zhigang Zhou et al (2015) } \\
\text { Hussein S. Al-Olimat et al(2015) }\end{array}$ \\
\hline & $\begin{array}{l}\text { Energy } \\
\text { Consumption }\end{array}$ & 7 & $\begin{array}{l}\text { A.Y.Khobragade et al (2013) } \\
\text { Wanneng Shu et al (2014) } \\
\text { Qi Zhang et al (2014) } \\
\text { Jayanthi S et al(2014) } \\
\text { Jing-Mian Tang et al(2015) } \\
\text { Devendra Singh Thakur et al } \\
\quad \text { Shilpi Saxena et al }\end{array}$ \\
\hline & $\begin{array}{l}\text { Cloud Computing } \\
\text { Environment }\end{array}$ & 3 & $\begin{array}{l}\text { Sagar.G et al (2013) } \\
\text { S.Thejeswi et al (2013) } \\
\text { S.Sujan et aL(2015) }\end{array}$ \\
\hline & $\begin{array}{l}\text { Advanced } \\
\text { Methodologies }\end{array}$ & 3 & $\begin{array}{l}\text { Chien-Hung Chen et al(2015) } \\
\text { D.A. Heger et al } \\
\text { Gunho Lee et al }\end{array}$ \\
\hline & Resource Allocation & 9 & $\begin{array}{l}\text { MS. Pooja .P.Vasani et al (2013) } \\
\text { Shivani Sharma et al(2014) } \\
\text { N.D. Doulamis et al(2014) } \\
\text { H.Wang et al(2015) } \\
\text { Longbin .C et al(2015) } \\
\text { A.Narayan Singh et al (2015) } \\
\text { Hui Wang et al(2015) } \\
\text { K.Beghdad Bey et al } \\
\text { Zhou Fang et al } \\
\text { K.Beghdad Bey et al }\end{array}$ \\
\hline & Virtual Machine & 3 & $\begin{array}{l}\text { Sharrukh .Z et al(2013) } \\
\text { Pratik P. Pandya et al(2014) } \\
\text { Omar Khedher et al(2015) }\end{array}$ \\
\hline & $\begin{array}{l}\text { Distributed Data } \\
\text { centers }\end{array}$ & 4 & $\begin{array}{l}\text { M.A.Sharkh et al(2013) } \\
\text { Shaolei Ren et al(2014) } \\
\text { Aziz Mohaisen et al(2014) } \\
\text { Seokho Son et al }\end{array}$ \\
\hline Scheduling & $\begin{array}{l}\text { Scheduling } \\
\text { Algorithm }\end{array}$ & & $\begin{array}{l}\text { Shaobin Zhan et al(2012) } \\
\text { Monika Choudhary et al(2012) } \\
\text { Upendra Bhoi et al(2013) } \\
\text { A.Kaleeswaran et al(2013) } \\
\text { Hilda Lawrance et al(2013) } \\
\text { Vijayalakshmi A et al(2013) }\end{array}$ \\
\hline
\end{tabular}




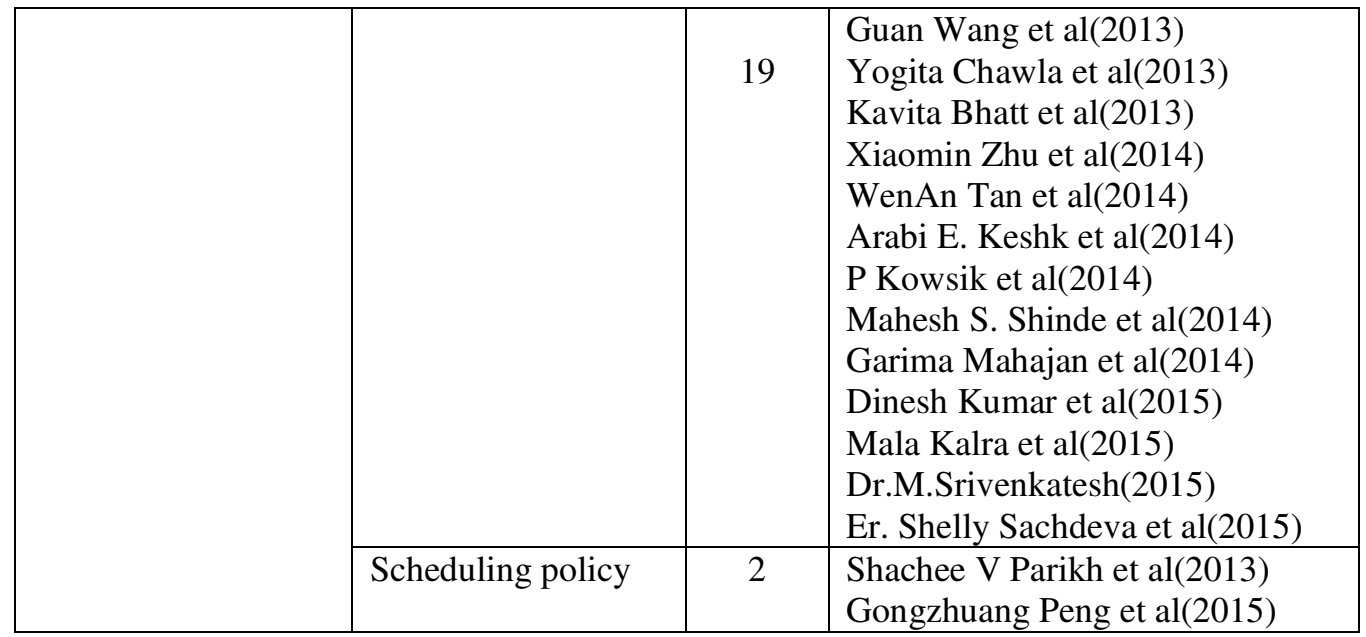

\section{OVERVIEW ON SYSTEMATIC LITERATURE REVIEW}

\subsection{Survey on Cloud Computing}

Haibo Yang et al. (2009) [10], Haibo Yang et al. (2012) [11], Sunilkumar S.M et al.(2013) [89], F.F.Moghaddam et al. (2015) [3], Mahmoud.M et al. (2014) [7], Nasrin Hesabian et al. (2015 [9], Anterpreet Kaur (2015) [22], K.Beghdad Bey et al. [84], MalaKalraetal(2015) [50] and Vijayalakshmi A et al.(2013) [70], authors are discussed on cloud computing technological issues such as network, performance, security, resource allocation, job scheduling, load balancing, data availability, interoperability, compatibility, scalability, and data management. The new theme of research works on social and organizational implications of cloud computing and the most important issue is related to security and privacy subjects in cloud-based environments. Furthermore, resource allocation and Job scheduling is one of the biggest issues in cloud computing for dedicating the resources to the system requests. Dedicating resources to the requests is a NP-complete problem due to requests and resource dynamics. One of the most pressing issues in cloud computing for IaaS is the resource management. Resource management problems include allocation, requirement provisioning, discovery, brokering, estimation, mapping, adaptation and modeling.

Yaser Ghanam et al. (2012) [12], Frederico Durao et al. (2014) [16], Anton Backe et al. (2015) [15], the authors are focusing on advances issues in the field of cloud computing such as security and privacy, resource sharing, billing and service layer agreement to opened up new questions about the real gains of the business model. The authors also suggested conducting intensive casestudies for understanding challenges related to design of software as well as testing the cloud environments.

Jaspreet kaur et al. (2012) [13], Lovejit Singh et al. (2013) [5], Sukhpal Singh et al. (2015) [2] [18], Soumen Santra et al. (2015) [4] authors are presented different strategies for efficient scheduling and resource allocation to decrease operational cost and time. These strategies solve the load distributing problem on various nodes of a distributed system to improve both resource utilization and job response time. It also avoiding a situation where some of the nodes are heavily loaded while other nodes are idle or doing very little work. Authors give a direction for selection of a particular scheduling algorithm depends upon various factors such as cost or time, quality of service, availability of information, etc. 
Shabnam Khan et al. (2013) [6] and A E Mohamed Mosa et al. (2014) [8] focused on the issue related to disk space management in virtual environment as well as amount of power consumption in data centers for which great amount of carbon dioxide emissions increases. Authors suggested that scheduling existing scheduling algorithms gives high throughput and cost effective but they don't considered availability and reliability issues.

To achieve good performance, high utilization of resources and less response time, Cloud computing required to execute many tasks by providing related resources and also for any type of complicated tasks in different environments of cloud is suggested by M.Padmavathi et al. (2014) [14] and Malathi.P et al. (2015) [17].

Yan Hu et al. (2014) [20] and Zhi-Hui Zhan et al.(2015) [1] discussed on three types of studies about cloud computing in eHealth, namely, cloud-based eHealth framework design, security or privacy control mechanisms of healthcare data in the cloud and applications of cloud computing. Li Liu et al. (2014) [21] and Rashmi Rai et al. (2015) [19] focused on the several critical issues such as maintainability, scalability, security, scale and complexity associated with the legacy system. Among the issues they considered security is prime factor for the adoption in cloud migration.

Ilango Sriram et al. (2015) [23] and Rahul Sharma et al. (2015) [24] are raised on different research challenges such as to facilitate industry in building successful clouds and comparing cloud computing features with pervasive as well as service computing.

\subsection{Survey on Resource Allocation}

Shubhangi D. et al.(2012) [38], Abirami S.P et al.(2012) [63], Ram Kumar Sharma et al.(2013) [72] , Xin Xu et al. (2014) [44], Zhigang Zhou et al. (2015) [35],Hussein S. Al-Olimat et al.(2015) [60] focuses on sharing the knowledge on utlization of resources in cloud computing environments such as cloud providers and cloud users with a minimal expense for which it meets performance requirements. The knowledge is applied on different services such as nimbus, cumulus and demand. N. Asha et al. (2013) [40] and Jayanthi S et al. (2014) [49] suggested on resource accounting and two distinct alternatives: one is strictly usage-oriented where user has a limited number of units, which can be connected to CPU and/or Memory usage, time. And another alternative is capacity pre-allocation.

A.Y.Khobragade et al. (2013) [39],Wanneng Shu et al. (2014) [26] and Jing-Mian Tang et al.(2015) [62] presented energy consumption offers in various computing utility services to the day to day life but the demand of Cloud infrastructure has greatly increased the energy consumption of data centers, which is a major big problem is facing the world today. It is very hard to evaluate and optimize energy consumption in complex cloud infrastructure architectures.

Devendra Singh Thakur et al. [83] and Shilpi Saxena et al. [85] discussed lot of challenges such as security of data, server consolidation, resources, tasks, data storage and consumption of energy. Qi Zhang et al. (2014) [30] and Zhou Fang et al. [47] said that the existing solutions have not fully considered the heterogeneity of both workload and machine hardware found in production environments. Failure to consider the heterogeneity of both machines and workloads will lead to both sub-optimal energy-savings and long scheduling delays, due to incompatibility between workload requirements and the resources offered by the provisioned machines.

Sagar.G et al. (2013) [41] and S.Thejeswi et al. (2013) [42] focuses on rapid development of cloud computing environment for mapping applications from large quantity of users. There must 
be a provision that all resources are dynamically made available to satisfy the needs of requesting users.

S.Sujan et al. (2015) [61] and H.Wang et al.(2015) [31]fixes the issue of computing millions of transactions a day by considering resource provisioning and scheduling context.

Chien-Hung Chen et al. (2015) [57], D. A. Heger et al. [46], Gunho Lee et al. [48] authors have given an idea on advanced methodologies used in cloud computing such as MapReduce scheduling and Data Analytics are required to assess the performance, capacity behavior and allocation with high fidelity.

Pooja .P.Vasani et al. (2013) [74] and Longbin .C et al. (2015) [36] are represented on different types of resources in virtualized form for dynamix utilization using scheduling.

N.D. Doulamis et al. (2014) [29], Christina Delimitrou et al. (2015) [52] and Hui Wang et al.(2015) [34] takeup a challenging issues called QoS (Quality of Service) for hybrid storage systems in resource allocation and also QoS degradation is expressed through time deviation metric.

Shivani Sharma et al. (2014) [80] and A.Narayan Singh et al. (2015) [32] sharing on various software and hardware resources for designing various resource allocation alogrithms in efficient manner.

Sharrukh .Z et al. (2013) [28], Pratik P. Pandya et al. (2014) [43] and Gruia Calinescu et al. (2011) [25] authors addressed the issue on dynamica provisioning VM instances in clouds to generate higher profit by considering various parameters like QoS, cost, time consumption, carbon effect. Omar Khedher et al. (2015) [59] have empowered cloud computing challenging task in the academic communities by reducing the IT infrastructure administration and cost by representing a simplified architecture design, treating a large size Virtual Cloud Laboratory (VCL) deployment.

Shaolei Ren et al. (2014) [55] and Seokho Son et al. [45] has discussed on wireless cloud computing services in which service providers keep deploying services in geographically distributed data centres and provides services based on dynamic price subscribe, Service Level Agreements to reduce the load on data centers.

M.A.Sharkh et al. (2013) [27] and Aziz Mohaisen et al. (2014) [88] has introduces Data Center Resource Allocation paradigms for internal and external challenges, computing nodes in social ties. The paradigms works based on bootstrapping trust-possessing social graph and these paradigms are different when compared with the paradigms of distributed, grid and conventional cloud computing.

\subsection{Survey on Scheduling}

Shaobin Zhan et al.(2012) [65], Kavita Bhatt et al.(2013) [71],WenAn Tan et al.(2014) [54], P Kowsik et al.(2014) [77], Garima Mahajan et al. (2014) [76] focused on job scheduling issues such as optimization algorithm, combination optimization,task schedulers, WFS-algorithms, workflow scheduling and load balancing.

Monika Choudhary et al.(2012) [64], Upendra Bhoi et al. (2013) [68], A. Kaleeswaran et al.(2013) [66],Hilda Lawrance et al.(2013) [67] discussed on static and dynamic parameters, 
restriction of tasks and resources achieving best performance such as CPU speed, memory, bandwidth, minimal total time for completion, shortest response time and utilization of resources .

Guan Wang et al.(2013) [87], Yogita Chawla et al.(2013) [69], Arabi E. Keshk et al.(2014) [75], Mahesh S. Shinde et al.(2014) [78], Dr.M.Srivenkatesh(2015) [81], Er. Shelly Sachdeva et al.(2015) [82] focused on task scheduling algorithms such as Min-min algorithm, conventional scheduling concepts. Task scheduling is divided in two types one is on-line mode and batch mode service.

,Xiaomin Zhu et al.(2014) [53] and Kapil Kumar er al.(2014) [79] presents energy conversation such as reducing operating costs, increasing system reliability, prompting environmental protection and energy -aware scheduling. Dinesh Kumar et. al. (2015) [56], discussed virtualization technology such as efficient virtual machine scheduling and physical machines.

Shachee V Parikh et al. (2013) [73], Antony Thomas et al. (2015) [51] and Gongzhuang Peng et al. (2015) [58] authors have addressed dynamic and heterogonous nature of resources and tasks in cloud computing by designing a framework based on scheduling policies. The framework monitors, detects and scheduling the system resource performance.

\section{DISSCUSION ON THE STUDY OF RESOURCE ALLOCATION AND SCHEDULING}

The main aim of the study is how research is moving drastically in different components of cloud computing such as Resource Utilization, Scheduling Algorithms, Resource Allocation, Scheduling Policies and Advanced Methodologies. Different issues such as Security, Privacy, Infrastructure, Data Management, Job Scheduling, Scalability, Time Complexity, Cost, Maintainability, Migration, Resource Optimization etc were discussed. At the outside, cost and resource efficiency is playing a vital role in IT organizations and it needs new technologies for business applications in cloud computing. To endeavor the security issue, one has to propose deployment methods and service models in cloud computing. To balance the load on cloud servers and to improve the performance as well as to enhance the resource and Job scheduling, new algorithms have to develop.

There is a research gap exists in: growing maturity of cloud migration with its evaluation, achieving interoperability, building successful industry clouds, managing virtual machine images, storing and processing features of cloud computing with pervasive computing and service computing, maximization of system utilization with minimizing make span to reduce cost, optimizing the algorithms to scale effectively with larger workloads, reducing energy consumptions, time span, revenue in single or multi-objective formulations.

Finally, we are planning to propose a model which deals with efficient allocation of the resources, analyze performance in the cloud system and also moving towards resource provisioning, task scheduling, deployment model and methods in cloud environment.

\section{CONCLUSION}

Cloud computing, the long-held dream of computing as a utility, has the potential to transform a large part of the IT industry, making software even more attractive as a service and shaping the way in which hardware is designed and purchased. Cloud Computing can be distinguished from other distributed paradigms though the provisioning of resources, data and software to users on demand in a similar fashion to the services provided by the electric power industry.

In the next work of research, we are going to deal with cloud environment, make cloud services and cloud-oriented applications efficiently. It works with task scheduling inside cloud computing, among them there is a particular interest in the ones caused by the influence of system volatility over the scheduling heuristics and in the problems caused by failures in some of the scheduling platform components. 


\section{REFERENCES}

[1] Zhi-Hui Zhan, Xiao-Fang Liu, Yue-Jiao Gong, Jun Zhang,Henry Shu-Hung Chung,Yun Li, (July 2015) "Cloud Computing Resource Scheduling and a Survey of Its Evolutionary Approaches," ACM Computing Surveys, Vol. 47, No. 4, Article 63.

[2] Sukhpal Singh and Inderveer Chana, (December 2015) "A Survey on Resource Scheduling in Cloud Computing: Issues and Challenges," Journal Grid Computing.

[3] Faraz Fatemi Moghaddam, Mohammad Ahmadi,Samira Sarvari,Mohammad Eslami and Ali Golkar, (2015) "Cloud Computing Challenges and Opportunities:A Survey," 1st International Conference on Telematics and Future Generation Networks(TAFGEN), pp.34-38.

[4] Soumen Santra and Dr. Kalyani Mali, (2015) "A New Approach to Survey on Load Balancing in VM in Cloud Computing: using CloudSim," IEEE International Conference on Computer, Communication and Control (IC4).

[5] Lovejit Singh and Sarbjeet Singh, (2013) "A Survey of Workflow Scheduling Algorithms and Research Issues", International Journal of Computer Applications, (74) (15), 21-28.

[6] Shabnam Khan, (2013) "A Survey On Scheduling Based Resource Allocation In Cloud Computing", International Journal For Technological Research In Engineering, (1) (1),.44-48.

[7] Mahmoud Maqableh, Huda Karajeh, Ra'ed Masa'deh, (August 2014) " Job Scheduling for Cloud Computing Using Neural Networks", Communications and Network, Vol. 6, pp.191-200.

[8] Abdelkhalik Elsaid Mohamed Mosa, (2014) "Adaptive Cloud Scheduling".

[9] Nasrin Hesabian And Hamid Haj Seyyed Javad, (2015) "Optimal Scheduling In Cloud Computing Environment Using The Bee Algorithm," International Journal Of Computer Networks And Communications Security, (3) (6), 253-258.

[10] Haibo Yang and Mary Tate,( Dec 2009) "Where are we at with Cloud Computing?: A Descriptive Literature Review," 20th Australasian Conference on Information Systems, pp.807-819.

[11] Haibo Yang and Mary Tate, (July 2012) "A Descriptive Literature Review and Classification of Cloud Computing Research," Communications of the Association for Information Systems,Vol. 31, Article 2, pp. 35-60.

[12] Yaser Ghanam, Jennifer Ferreira and Frank Maurer, (2012) "Emerging Issues \& Challenges in Cloud Computing-A Hybrid Approach," Journal of Software Engineering and Applications, (5), 923937.

[13] Jaspreet kaur, (2012) "Comparison of load balancing algorithms in a Cloud," International Journal of Engineering Research and Applications(IJERA), (2) (3) ,1169-1173.

[14] M.Padmavathi, Shaik. Mahabbob Basha and Srinivas Pothapragada, (2014) "A Survey on Scheduling Algorithms in Cloud Computing," Journal of Computer Engineering (IOSR-JCE), (16) (4), 27-32.

[15] Anton Backe and Hugo Linden, (2015) "Cloud Computing Security:A Systematic Literature Review".

[16] Frederico Durao,Jose Fernando S, Carvalho,Anderson Fonseka,Vinicius Cardoso Garcia, (January 2014) "A systematic review on cloud computing," Journal of Super computer.

[17] Malathi.P And Arumugam.S, (2015) "A Survey: To Harness An Efficient Energy In Cloud Computing," International Journal Of Ubicomp (Iju), (6) (3), 1-7.

[18] Sukhpal Singh And Inderveer Chana, (December 2015) "QoS-Aware Autonomic Resource Management in Cloud Computing: A Systematic Review," ACM Computing Surveys, (48) (3-42), 110.

[19] Rashmi Rai, Gadadhar Sahoo and Shabana Mehfuz, (2015) "Exploring the factors influencing the cloud computing adoption: a systematic study on cloud migration," SpringerPlus.

[20] Yan Hu And Guohua Bai, (2014) "A Systematic Literature Review Of Cloud Computing In Ehealth," Health Informatics-An International Journal (HIIJ), (3) (4), 11-20.

[21] Li Liu,Miao Zhang,Yuqing Lin and Liangjuan Qin, (2014) "A Survey on Workflow Management and Scheduling in Cloud Computing," 14th IEEE/ACM International Symposium on Cluster, Cloud and Grid Computing, pp.837-846.

[22] Anterpreet Kaur, (2015) "A Review of Workflow Scheduling in Cloud Computing Environment," International Journal of Computer Science Engineering (IJCSE), (4) (2), 47-50.

[23] Ilango Sriram and Ali Khajeh-Hosseini, "Research Agenda in Cloud Technologies," (unpublished). 
International Journal of Advanced Information Technology (IJAIT) Vol. 6, No.4, August 2016

[24] Rahul Sharma And Samandeep Singh, (2015) "Current Research In Cloud Computing: A Review," International Journal Of Advanced Engineering Technology, (6) (2).

[25] Gruia Calinescu, Amit Chakrabarti, Howard Karloff, Yuval Rabani, (September 2011) "An Improved Approximation Algorithm For Resource Allocation," ACM Transactions On Algorithms, (7) (4-48).

[26] Wanneng Shu, Wei Wang and Yunji Wang, (2014) "A novel energy-efficient resource allocation algorithm based on immune clonal optimization for green cloud computing," EURASIP Journal on Wireless Communications and Networking.

[27] Mohamed Abu Sharkh, Manar Jammal, Abdallah Shami, and Abdelkader Ouda, (November 2013) "Resource Allocation in a Network-Based Cloud Computing Environment: Design Challenges," IEEE Communications Magazine.

[28] Sharrukh Zaman,and Daniel Grosu, (July-December 2013) "A Combinatorial Auction-Based Mechanism for Dynamic VM Provisioning and Allocation in Clouds," IEEE Transactions On Cloud Computing, (1) (2).

[29] Nikolaos D. Doulamis, Panagiotis Kokkinos, and Emmanouel (Manos) Varvarigos, (February 2014) "Resource Selection for Tasks with Time Requirements Using Spectral Clustering," IEEE Transactions On Computers, (63) (2).

[30] Qi Zhang, Mohamed Faten Zhani, Raouf Boutaba and Joseph L. Hellerstein, (January-March 2014) "Dynamic Heterogeneity-Aware Resource Provisioning in the Cloud," IEEE Transactions On Cloud Computing, (2) (1).

[31] Haiyang Wang, Feng Wang, Jiangchuan Liu,Dan Wang and Justin Groen, (July 2015) "Enabling Customer-Provided Resources for Cloud Computing: Potentials, Challenges, and Implementation," IEEE Transactions On Parallel And Distributed Systems, (26) (7).

[32] Aditya Narayan Singh and Shiva Prakash, (2015) "Challenges and Opportunities of Resource Allocation in Cloud Computing: A Survey," 2nd International Conference on Computing for Sustainable Global Development (INDIACom), pp.2047-2051.

[33] Kadda Beghdad Bey,Farid Benhammadi,Faouzi Sebbak and Mhamed Mataoui, "New tasks scheduling strategy for resources allocation in Cloud Computing Environment,".

[34] Hui Wang and Peter Varman, (2015) "A Resource Allocation Model for Hybrid Storage Systems," 15th IEEE/ACM International Symposium on Cluster, Cloud and Grid Computing.

[35] Zhigang Zhou, Hongli Zhang, Xiangzhan Yu, and Junwu Guo, (2015) "Continuous Resource Allocation in Cloud Computing," IEE ICC SAC-Data Storage and Cloud Computing, pp.319-324.

[36] Longbin Chen, Yucong Duan. Meikang Qiu, Jian Xiong, Keke Gai, (2015) "Adaptive Resource Allocation Optimization in Heterogeneous Mobile Cloud Systems," IEEE 2nd International Conference on Cyber Security and Cloud Computing, pp.19-24.

[37] Ikki Fujiwara, (March 2012) "Study on Combinatorial Auction Mechanism for Resource Allocation in Cloud Computing Environment,".

[38] Ms. Shubhangi D. Patil and Dr. S. C. Mehrotra, (2012) "Resource Allocation and Scheduling in the Cloud," International Journal of Emerging Trends and Technology in Computer Science (IJETTCS), (1) (1), 47-52.

[39] Apeksha Yashpal Khobragade and Jeya Krishnan, (2013) "A Novel Approach for Energy Aware Resource Allocation Using Hybrid Heuristics in Cloud Computing," International Journal of Computer Science and Management Research, (2) (1),1385-1391.

[40] N. Asha and Dr. G. Raghavendra Rao, (2013) "A Review on Various Resource Allocation Strategies in Cloud Computing," International Journal of Emerging Technology and Advanced Engineering(IJETAE), (3) (7), 117-183.

[41] Sagar Girase, Rahul Samant, Mayank Sohani and Suraj Patil, (2013) "Review on:Resource Provisioning in Cloud Computing Environment," International Journal of Science and Research (IJSR), (2) (11), 337-341.

[42] S.Thejeswi and C.Thirumalaiselvan, (2013) "Resource Allocation In Self Organizing Cloud - A SURVEY," International Journal of Computer Networks and Wireless Communications (IJCNWC), (3) (6), 423-432 .

[43] Pratik P. Pandya and Hitesh A. Bheda, (2014) "Dynamic Resource Allocation Techniques in Cloud Computing," International Journal of Advance Research in Computer Science and Management Studies, (2) (1), 559-563.

[44] Xin Xu and Huiqun Yu, (April 2014) "A Game Theory Approach to Fair and Efficient Resource Allocation in Cloud Computing," Mathematical Problems in Engineering , pp.7-14. 
International Journal of Advanced Information Technology (IJAIT) Vol. 6, No.4, August 2016

[45] Seokho Son, Gihun Jung, and Sung Chan Jun, "A SLA-based Cloud Computing Framework: Workload and Location Aware Resource Allocation to Distributed Data Centers in a Cloud," (unpublished).

[46] Dominique A. Heger, "Optimized Resource Allocation and Task Scheduling Challenges in Cloud Computing Environments," (unpublished).

[47] Zhou Fang, Buyang Cao And Xu Jiang, "A Research On Resource Allocation Solution With Cost Optimization In Cloud Computing System," (unpublished).

[48] Gunho Lee, Byung-Gon Chun, Randy H. Katz, "Heterogeneity-Aware Resource Allocation and Scheduling in the Cloud," (unpublished).

[49] Jayanthi S, (2014) "Literature Review: Dynamic Resource Allocation Mechanism in Cloud Computing Environment," International Conference on Electronics, Communication and Computational Engineering (ICECCE), pp.279-281.

[50] Mala Kalra, Sarbjeet Singh , (july 2015) "A review of metaheuristic scheduling techniques in cloud computing," Egyptian Informatics Journal.

[51] Antony Thomas , Krishnalal G, Jagathy Raj V P, (December 2015) "Credit Based Scheduling Algorithm in Cloud Computing Environment," in Proc of Computer Science, Vol. 46, pp.913 - 920.

[52] Christina Delimitrou And Christos Kozyrakis, (December 2013) "QoS-Aware Scheduling in Heterogeneous Datacenters with Paragon," ACM Transactions on Computer Systems, (31) (4-12).

[53] Xiaomin Zhu, Laurence T. Yang, Huangke Chen,Ji Wang, Shu Yin and Xiaocheng Liu, (April-June 2014) "Real-Time Tasks Oriented Energy-Aware Scheduling in Virtualized Clouds," IEEE Transactions On Cloud Computing, (2) (2).

[54] WenAn Tan, Yong Sun, Ling Xia Li, GuangZhen Lu, and Tong Wang, (2014) "A Trust ServiceOriented Scheduling Model for Workflow Applications in Cloud Computing," IEEE Systems Journal, (8) (3).

[55] Shaolei Ren and Mihaela van der Schaar, (October 2014) "Dynamic Scheduling and Pricing in Wireless Cloud Computing," IEEE Transactions On Mobile Computing, (13) (10).

[56] Dinesh Kumar and Zahid Raza, (2015) "A PSO based VM Resource Scheduling Model for Cloud Computing," IEEE International Conference on Computational Intelligence and Communication Technology.

[57] Chien-Hung Chen, Jenn-Wei Lin, and Sy-Yen Kuo, (2015) "MapReduce Scheduling for DeadlineConstrained Jobs in Heterogeneous Cloud Computing Systems," IEEE Transactions on Cloud Computing.

[58] Gongzhuang Peng, Jiaxin Zhao, Minghui Li,Baocun Hou and Heming Zhang, (2015) "A SLA-based Scheduling Approach for Multi-tenant Cloud Simulation," IEEE 19th International Conference on Computer Supported Cooperative Work in Design (CSCWD).

[59] Omar Khedher,Mohamed Jarraya, (2015) "Improved Scheduling Algorithm In VCL Cloud Computing Environment On CloudSim," IEEE, pp.254-261.

[60] Hussein S. Al-Olimat,Mansoor Alam,Robert Green and Jong Kwan Lee, (2015) "Cloudlet Scheduling with Particle Swarm Optimization," 5th International Conference on Communication Systems and Network Technologies, pp.991-995.

[61] S.Sujan and R.Kanniga Devi, (2015) "A Batchmode Dynamic Scheduling Scheme For Cloud Computing," Global Conference on Communication Technologies(GCCT), pp.297-302.

[62] Jing-Mian Tang,Liang Luo, Kai-Ming Wei,Xun Guo,Xiao-Yu Ji, (2015) "A heuristic resource scheduling algorithm of cloud computing based on polygons correlation calculation," IEEE 12th International Conference on e-Business Engineering, pp.365-370.

[63] Abirami S.P. and Shalini Ramanathan, (2012) "Linear Scheduling Strategy for Resource Allocation in Cloud Environment," International Journal on Cloud Computing: Services and Architecture(IJCCSA), (2) (1), 9-17.

[64] Monika Choudhary and Sateesh Kumar Peddoju, (2012) "A Dynamic Optimization Algorithm for Task Scheduling in Cloud Environment," International Journal of Engineering Research and Applications (IJERA), (2) (3), 2564-2568.

[65] Shaobin Zhan and Hongying Huo, (2012) "Improved PSO-based Task Scheduling Algorithm in Cloud Computing," Journal of Information \& Computational Science , (9) (13), 3821-3829.

[66] A.Kaleeswaran, V.Ramasamy and P.Vivekanandan, (2013) "Dynamic Scheduling Of Data Using Genetic Algorithm In Cloud Computing," International Journal of Advances in Engineering \& Technology(IJAET), (5) (2), 327-334. 
[67] Hilda Lawrance and Dr. Salaja Silas, (2013) "Efficient Qos Based Resource Scheduling Using PAPRIKA Method for Cloud Computing," International Journal of Engineering Science and Technology (IJEST), (5) (3), 638-643.

[68] Upendra Bhoi and Purvi N. Ramanuj, (2013) "Enhanced Max-min Task Scheduling Algorithm in Cloud Computing," International Jouranl of Application or Innovation in Engineering \& Management(IJAIEM), (2) (4), 259-264.

[69] Yogita Chawla and Mansi Bhonsle, (2013) "Dynamically optimized cost based task scheduling in Cloud Computing," International Journal of Emerging and Technology in Computer Science (IJETTCS), (2) (3), 38-42.

[70] Vijayalakshmi A. Lepakshi and Dr. Prashanth C S R, (2013) "A Study on Task Scheduling Algorithms in Cloud Computing," International Journal of Engineering and Innovative Technology (IJEIT), (2) (11), 119-125.

[71] Kavita Bhatt and Dr. Mahesh Bundele, (2013) "Review Paper on PSO in workflow scheduling and Cloud Model enhancing Search mechanism in Cloud Computing," International Journal of Innovations in Engineering and Technology (IJIET), (2) (3), 68-74.

[72] Ram Kumar Sharma and Nagesh Sharma, (2013) "A Dynamic Optimization Algorithm for Task Scheduling in Cloud Computing With Resource Utilization," International Journal of Scientific Engineering and Technology(IJSET), (2) (10), 1062-1068.

[73] Shachee V Parikh And Assis Prof Richa Sinha, (2013) "Double Level Priority Based Task Scheduling With Energy Awareness In Cloud Computing," Journal Of Information, Knowledge And Research In Information Technology(JIKRIT), (2) (2), 142-147.

[74] Ms. Pooja ,P.Vasani, Mr. Nishant And S. Sanghani, (2013) "Literature Review: Various Priority Based Task Scheduling Algorithms In Cloud Computing," Journal Of Information, Knowledge And Research In Computer Engineering, (2) (2), 298-302.

[75] Arabi E. Keshk, (2014) "Cloud Computing Online Scheduling," International organization of Scientific Research(IOSR), (4) (3), 7-17.

[76] Garima Mahajan, (2014) "Job Scheduling In Cloud Computing: A Review of Selected Techniques," International Journal of Emerging Trends and Technology in Computer Science (IJETTCS), (3) (3), 253-257.

[77] P.Kowsik and K.Rajakumari, (2014) "A Comparative Study on Various Scheduling Algorithms in Cloud Environment," International Journal of Innovative Research in Computer and Communication Engineering, (2) (11), 6494-6500.

[78] Mahesh S. Shinde and Anilkumar Kadam, (2014) "Survey of Recent Task Scheduling Strategies in Cloud Computing," International Journal of Advanced Research in Computer Science and Software Engineering,(4) (11), 830-834.

[79] Kapil Kumar, Abhinav Hans, Ashish Sharma and Navdeep Singh, (November 2014) "Towards The Various Cloud Computing Scheduling Concerns: A Review," International Conference on Innovative Applications of Computational Intelligence on Power, Energy and Controls with their Impact on Humanity (CIPECH14),pp.482-485.

[80] Shivani Sharma and Dhanshri Parihar, (2014) "A Review on Resource Allocation in Cloud Computing," International Journal of Advance research, Ideas and Innovations in Technology, (1) (3).

[81] Dr.M.Srivenkatesh and K,Vanitha, (2015) "Task Scheduling in Federated Cloud by Multilevel Queue based on Genetic Algorithm," International Journal of Advanced Research in Computer and Communication Engineering , (4) (3), 440-444.

[82] Shelly Sachdeva,Meenakshi Sharma and Lavina Maheshwari, (2015) "A Review on: Task Scheduling in Cloud Computing," International Journal of Advanced Research in Computer Science and Software Engineering, (5) (8), 685-690.

[83] DevendraSinghThakur, "Energy Efficient Task Scheduling Algorithms In Cloud Data Center," (unpublished).

[84] Kadda Beghdad Bey,Farid Benhammadi and Rédha Benaissa, "Balancing Heuristic for Independent Task Scheduling in Cloud Computing," (unpublished).

[85] Shilpi Saxena,Deepika Saxena, "EWSA: An Enriched Workflow Scheduling Algorithm in Cloud Computing,"

[86] Vijay Prakash, "An Efficient Workflow Scheduling Approach in Cloud Computing," (unpublished).

[87] Guan Wang and Haicun Yu, (Feb 2013) "Task scheduling algorithm based on improved Min-Min algorithm in cloud computing environment," Applied Mechanics and Materials,Vols. 303306,pp.2429-243. 
International Journal of Advanced Information Technology (IJAIT) Vol. 6, No.4, August 2016

[88] Aziz Mohaisen,Huy Tran, Abhishek Chandra and Yongdae Kim, (July-September 2014) "Trustworthy Distributed Computing on Social Networks," IEEE Transactions On Services Computing, Vol. 7, No. 3.

[89] SunilkumarS.Manvi,GopalKrishnaShyam, (October 2013) "Resource management for Infrastructure as a Service(IaaS) in cloud computing: Asurvey," Journal of Network and ComputerApplications.

Authors

Smt. B. Muni Lavanya is currently working as Adhoc Lecturer at JNTUA College of Engineering, Pulivendula. She completed her B.Tech Degree in Computer Science and Engineering from Sree Vidyanikethan Engineering College, Tirupathi in 2009. She completed her M.Tech Degree in Computer Science and Engineering from JNT University, Anantapur in 2012. She is pursuing Ph. D. from JNT University Anantapur. Her specializations are: Big Data, Cloud Computing, Parallel Processing.

Dr. C .Shoba Bindu, received her Ph.D degree in Computer Science and Engineering from JNTUA, Anantapuramu, Andhra Pradesh. She is currently extending her services as Associate Professor in department of Computer Science and Engineering, JNTUA. She has guided many external and internal projects and has good contributions in many reputed journals. Her research interests are in the areas of Mobile and Adhoc Networks, Network security, Data Mining and Cloud Computing.
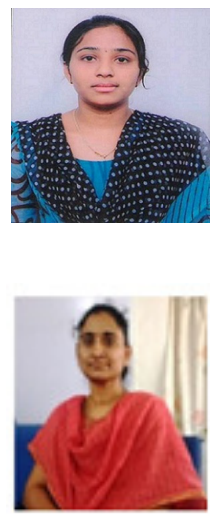